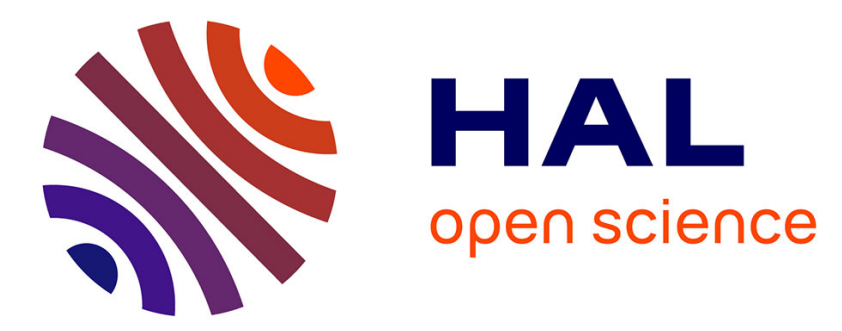

\title{
Relaxation magnétique d'atomes de rubidium sur des parois paraffinées
}

\author{
Marie-Anne Bouchiat
}

\section{To cite this version:}

Marie-Anne Bouchiat. Relaxation magnétique d'atomes de rubidium sur des parois paraffinées. Journal de Physique, 1963, 24 (6), pp.379-390. 10.1051/jphys:01963002406037900 . jpa-00205490

\section{HAL Id: jpa-00205490 https://hal.science/jpa-00205490}

Submitted on 1 Jan 1963

HAL is a multi-disciplinary open access archive for the deposit and dissemination of scientific research documents, whether they are published or not. The documents may come from teaching and research institutions in France or abroad, or from public or private research centers.
L'archive ouverte pluridisciplinaire HAL, est destinée au dépôt et à la diffusion de documents scientifiques de niveau recherche, publiés ou non, émanant des établissements d'enseignement et de recherche français ou étrangers, des laboratoires publics ou privés. 


\title{
RELAXATION MAGNÉTIQUE D'ATOMES DE RUBIDIUM SUR DES PAROIS PARAFFINÉES
}

\author{
Par Marie-Anne BOUChiAT, \\ Laboratoire de Physique de l'École Normale Supérieure, Paris.
}

\begin{abstract}
Résumé $\left({ }^{1}\right)$. - Les résultats expérimentaux montrent que la relaxation d'atomes de Rubidium orientés (à la pression de $10^{-7} \mathrm{~mm}$ de mercure et en l'absence de gaz tampon) se fait sur la paroi. Il y a, en outre, des raisons de penser que si la paroi est enduite de paraffine, l'interaction prépondérante qui détermine le phénomène de relaxation se produit entre les moments magnétiques de l'électron de valence du Rubidium (spin S) et ceux des protons (ou deutons) de la paraffine (spins $K$ ). Nous donnons ci-après l'étude théorique de ce mécanisme de relaxation. Le calcul peut se faire dans le cadre du modèle dit avec " moyennage par le mouvement ". Les spins S sont soumis, par ailleurs, à deux interactions statiques : l'une est le couplage aS.I de $S$ avec le spin nucléaire $I$ de. l'alcalin, l'autre est l'interaction Zeeman avec le champ magnétique statique.

Dans la première partie (qui figure seule dans le présent article), nous envisageons le cas d'un champ faible (interaction hyperfine grande devant l'effet Zeeman) ; nous calculons l'équation d'évolution de la matrice densité et de plusieurs observables : population d'un niveau hyperfin, aimantations longitudinales et transversales électroniques et nucléaires. Dans la $2^{\mathrm{e}}$ puis la $3^{\mathrm{e}}$ partie (qui feront l'objet d'un second article), nous envisageons le cas d'un champ très fort puis d'un champ quelconque. Dans la $4^{\mathrm{e}}$ partie, nous généralisons nos résultats au cas où l'interaction responsable de la relaxation n'agit sur l'atome de $\mathrm{Rb}$ que par l'intermédiaire de son spin électronique $S$. Cette étude est nécessaire pour l'interprétation des mesures expérimentales relatives à la détermination du temps de corrélation d'une interaction de ce type.
\end{abstract}

\begin{abstract}
Experiments on polarized Rubidium atoms (without buffer gas and at a pressure of $10^{-7} \mathrm{mmHg}$ ) show that relaxation takes place on the walls ; furthermore, they suggest that the relaxation mechanism is mostly to be found in the dipolar coupling beetween the valence electron of Rubidium (spin S) and protons (or deutons) of the walls (spin $K$ ). We analyze here theoretically this relaxation process. The "motion narrowing" model can be used. Two different static interactions act on spins $S$ : first, the hyperfine coupling a $S . I$ (I, nuclear spin of the alkali), and second, the Zeeman interaction with the d. c. magnetic field.

In the first part of this work (the only one included in the present paper) we consider the case of a small magnetic field (hyperfine splitting large compared to Zeeman splitting); we derive differential equations describing the rate of change for the $\mathrm{Rb}$ atoms density matrix and for several observables as population of a hyperfine level, longitudinal, transversal, electronic and nuclear polarizations. In the second and the third parts of our work (which will be published in a second article), we consider the case of a strong field and then of a field of any value. In the fourth part, we show that our results are still valid for all interactions acting only on $S$. This study is necessary to get an interpretation of experiments relative to the determination of the correlation time for such interactions.
\end{abstract}

Introduction. - Nóus avons effectué une étude expérimentale de la relaxation longitudinale d'atomes de Rubidium orientés optiquement lors des collisions sur des parois recouvertes d'enduits de paraffines saturées. Les conditions expérimentales et les résultats correspondants ont été décrits en détail [1 et 2]. Nous rappelons seulement ici les faits suivants :

1) Les cellules ne contiennent pas de gaz tampon. La pression de vapeur de Rubidium est assez faible pour que l'effet des collisions $\mathrm{Rb}-\mathrm{Rb}$ soit négligeable.

2) Les mesures ont porté sur la relaxation de $\left\langle S^{z}\right\rangle$, S étant le spin électronique de l'atome de $\mathrm{Rb}$.

(1) Un second article faisant suite à celui-ci sera publié dans un prochain numéro du Journal de Physique.

(2) Il en est ainsi pour la relaxation de ${ }^{87} \mathrm{Rb}$ sur des enduits hydrogénés. Par contre, nous avons observé une déviation par rapport à une exponentielle unique pour la relaxation de ${ }^{87} \mathrm{Rb}$ sur des enduits deutérés,
3) $<S_{z}>$ relaxe exponentiellement avec une constante de temps $\left.T_{1}{ }^{2}\right)$, et $T_{1}$ est proportionnel au diamètre de la cellule. L'interaction désorientatrice se produit donc durant le temps de séjour $\tau_{\mathrm{s}}$ de l'atome de $\mathrm{Rb}$ sur la paroi, et $\tau_{\mathrm{s}}$ (temps d'interaction) est court devant le temps de vol $\tau_{\mathrm{v}}$, (temps moyen qui sépare deux collisions successives du même atome de $\mathrm{Rb}$ sur les parois de la cellule). $L e$ temps de corrélation de l'interaction désorientatrice $\tau_{\mathrm{c}}$ est lui-même, au plus égal à $\tau_{\mathrm{s}}$.

4) Les valeurs mesurées pour $T_{1}$ (et $T_{2}$ ) sont très longues par rapport à $\tau_{\mathrm{v}}$ : des milliers de collisions se produisent sans que l'orientation du Rubidium soit affectée. Nous avons donc affaire à des collisions faibles satisfaisant à la condition de rétrécissement par le mouvement : l'étude théorique de la relaxation peut se faire dans le cadre du modèle dit "avec moyennage par le mouvement" comme pour les liquides et les gaz [3]. Comme il est bien connu, les résultats s'expriment en fonction de $\omega$ (distances des niveaux d'énergie de l'atome 
de Rubidium) ${ }^{1}$ ), de $\tau_{c}$ et des carrés des éléments de matrice de l'interaction désorientatrice.

La perturbation ayant un spectre de Fourier de " largeur », 1/ $\tau_{c}$, elle n'est susceptible d'induire des transitions entre les sous-niveaux de l'état fondamental de l'atome de Rubidium (c'est-à-dire de le désorienter) que si $\omega$ est petit par rapport à $1 / \tau_{c}$, c'est-à-dire lorsque $\omega \tau_{\mathrm{c}} \ll 1$. Dans le cas contraire la fréquence $\omega$ est pratiquement absente du spectre de l'interaction désorientatrice qui est alors très inefficace pour relaxer les spins.

On peut faire varier $\omega$ en changeant le champ magnétique statique $H_{0}$. L'étude de la variation de $T_{1}$ en fonction de $H_{0}$ permet la détermination du temps de corrélation $\tau_{\mathrm{c}}$ et par suite d'une limite inférieure de $\tau_{\mathrm{s}}$.

5) On observe que $T_{1}$ est pratiquement le même quelle que soit la longueur de la chaîne de la paraffine hydrogénée constituant l'enduit et que $T_{1}$ est 4 à 6 fois plus long pour une paraffine deutérée; (le moment magnétique nucléaire de ${ }^{2} \mathrm{H}$ est 3 fois plus petit que celui de ${ }^{1} \mathrm{H}$; celui du carbone est nul). Ceci nous a conduit à penser qu'une partie au moins de la relaxation sur la paroi se fait entre le moment magnétique électronique $\mu_{s} \mathrm{du} R \mathrm{Rb}$ et les moments magnétiques nucléaires $\boldsymbol{\mu}_{K}$ des atomes (d'hydrogène ou de deutérium) de l'enduit (leur paramagnétisme électronique étant bloqué dans une liaison chimique).

C'est ce type d'interaction dont nous présentons ici l'étude théorique. Un calcul détaillé de ce type est donné dans le livre d'Abragam [3] au chapitre de la relaxation thermique dans les liquides et les gaz; il concerne le cas de 2 ensembles de spins $S$ et $K$ qui interagissent d'une façon aléatoire par l'intermédiaire de leurs moments magnétiques. Dans le cas traité ici, le spin que nous observons $S(S=1 / 2)$ appartient à un atome (Rb) ayant une structure hyperfine : Outre l'interaction avec le champ magnétique $H_{0}$, les spins $S$ sont donc soumis au couplage statique avec un autre spin I, couplage représenté par le Hamiltonien aS.I (par suite, en champ magnétique nul, le niveau fondamental de l'atome se compose de 2 niveaux hyperfins $F_{ \pm}=I \pm 1 / 2$ dont l'écart d'énergie est $\Delta W$ ). Ce caractère modifie sensiblement les résultats de la référence 3 : d'une part les temps de relaxation peuvent changer d'ordre de grandeur suivant la multiplicité du spin I; d'autre part les variations de $T_{1}$ et $T_{2}$ en fonction du champ $H_{0}$ sont très affectées par l'existence de $I$, car la grandeur relative des deux termes du hamiltonien statique dépend de $H_{0}$. L'interprétation des variations de $T_{1}, T_{2} \ldots$ en fonction $\mathrm{du}$ champ est donc complexe : Or, elle est importante puisqu'elle permet d'évaluer l'ordre de grandeur du temps de corrélation $\tau_{c}$ ou d'établir l'existence de plusieurs interactions de temps de corrélation

(1) Nous exprimons toujours par la suite les énergies en unités de pulsation. différents : $\tau_{c}, \tau_{c}^{\prime} \ldots$ et par suite une limite inférieure de $\tau_{\mathrm{s}}$. Ces faits justifient les calculs présentés ci-après.

Hypothèses Du calgul. - Les hypothèses du calcul sont les suivantes :

1) Entre deux collisions le spin $S$ est soumis uniquement au hamiltonien statique $\mathfrak{H}_{0}(S)$

$$
\mathfrak{H}_{0}(S)=a S . I .+\omega_{S} S_{z}-\omega_{I} I_{z} .
$$

2) Dans les expériences réalisées jusqu'ici et que nous avons rappelées, nous mesurons la polarisation électronique $\left\langle S_{z}\right\rangle$ des atomes de rubidium ; une méthode tout à fait analogue doit permettre d'obtenir un signal mesurant la différence de population entre les 2 sous-niveaux hyperfins $F_{ \pm}$; de même nous projetons l'étude de l'évolution de $\left\langle S^{+}\right\rangle$sous l'effet de la relaxation. Mais en aucun cas, nous n'introduisons (par un champ de radiofréquence) de "cohérence " [13] entre deux sous-niveaux hyperfins différents. Aussi, tout au long du calcul, nous nous bornons à envisager le cas où la matrice densité de l'ensemble des atomes de rubidium est diagonale en $F$, (mais pas nécessairement en $m_{\mathrm{F}}$ ). Soit un élément de matrice :

$$
\sigma_{F m_{\mathrm{F}}}^{F^{\prime} m_{\mathrm{F}}^{\prime}}=\overline{a_{F m_{\mathrm{F}}} a_{F^{\prime} m_{\mathrm{F}}^{\prime}}}
$$

$a_{F m_{\mathrm{F}}}, a_{F^{\prime} m_{\mathrm{F}}^{\prime}}$ représentent les coefficients $\mathrm{du}$ développement de la fonction d'onde d'un atome de $\mathrm{Rb}$ suivant les états propres $\left|F m_{\mathrm{F}}>,\right| F^{\prime} m_{\mathrm{F}}^{\prime}>$; la moyenne porte sur tous les atomes. L'hypothèse précédente signifie que $\sigma_{F m_{F}}^{F^{\prime} m^{\prime}}$ n'est différent de zéro que si $F=F^{\prime}$. Cette hypothèse a bien un sens car si un état ne possède pas initialement de " cohérence " entre des niveaux hyperfins différents, il ne peut en acquérir sous l'effet de la relaxation, phénomène aléatoire d'un atome à l'autre et sans oorrélation. Nous aurons l'occasion de vérifier ce point sur nos équations.

Il est clair que l'étude de l'évolution de la cohérence hyperfine sous l'effet de la relaxation peut se faire dans le cadre du formalisme exposé ci-dessous, mais nous ne l'abordons pas ici.

3) Nous admettons que lors d'une collision, le temps de séjour sur la paroi est déterminé par une force (type Van der Walls par exemple) ne dépendant pas du spin électronique et qui ne produit pas de désorientation.

4) L'interaction responsable de la relaxation se fait entre les moments magnétiques $\mu_{S}$ de l'électron de valence d'un atome $R b$ et $\boldsymbol{\mu}_{K}$ du noyau d'un atome de l'enduit. Nous montrerons dans la dernière partie de ce travail que les résultats obtenus se généralisent au cas où l'interaction possède uniquement la propriété suivante : elle $n$ 'agit sur la fonction d'onde de l'atome de Rb que par l'intermédiaire du spin électronique, S. (C'est le cas, en particulier, pour l'interaction décrite par Bernheim, entre le spin $S$ 
et les courants résultant de la collision sur la paroi [4].)

5) Soit $\mathfrak{H}_{1}(t)$ le hamiltonien perturbateur. La fraction $\frac{\tau_{\mathrm{S}}}{\tau_{\mathrm{S}}+\tau_{\mathrm{V}}}$ des atomes se trouve à l'instant $t$ sur la paroi. Pour cette fraction d'atomes, nous admettons que la fonction de corrélation de la perturbation est :

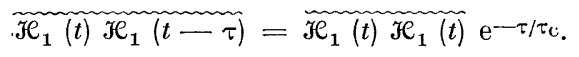

Pour l'ensemble de tous les atomes de rubidium, nous avons

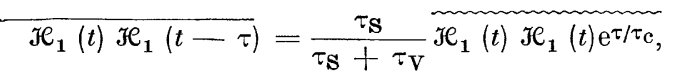

(le trait ondulé au-dessus du produit $\mathfrak{H}_{1}(t)$ $\mathfrak{H}_{1}(t-\tau)$ signifie que la moyenne est prise sur la fraction des atomes qui, à l'instant $t$, se trouvent sur la paroi et le trait droit, qu'elle est prise sur l'ensemble de tous les atomes.

$\widetilde{H_{1}}(t) \mathscr{H}_{1}(t)$ est un opérateur indépendant du temps. Comme nous l'avons déjà signalé, l'expé-

rience justifie l'hypothèse $\overline{\mathfrak{H}_{1}(t)} \mathscr{H}_{1}(t) \quad \tau_{\mathrm{c}}^{2} \ll 1$ nécessaire à la théorie du moyennage par le mouvement.

6) Nous admettons que l'orientation des spins $K$ de la paroi varie d'un point à un autre de la surface de façon aléatoire. Ceci revient à dire que si l'on prend la moyenne sur un grand nombre de spins $K$ à un instant $t$ donné, les composantes $K^{(r)}$

$$
K^{(0)}=K_{z}, \quad K^{( \pm 1)}=\frac{1}{\sqrt{2}}\left(K_{x} \pm i K_{y}\right),
$$

satisfont à la relation :

$\overline{K^{(r)}(t) K^{\left(r^{\prime}\right)}(t)}=\overline{\frac{1}{3}|K(t)|^{2}} \delta_{r,-r^{\prime}}=\frac{K(K+1)}{3} \delta_{r,-r^{\prime}}$

On peut justifier cette hypothèse en faisant les remarques suivantes : Le nombre des spins $S$ est très petit devant celui des spins $K$ : l'expérience montre en effet que $\tau_{S}$ est de l'ordre de $10^{-10}$ sec., si bien que la proportion de spins $S$ qui se trouvent sur la paroi est :

$$
\tau_{\mathrm{S}} /\left(\tau_{\mathrm{S}}+\tau_{\mathrm{v}}\right) \sim 10^{-6}
$$

Tous les spins $S$ sont donc pratiquement en phase vapeur. Si l'on opère avec une pression de Rb de $10^{-7} \mathrm{~mm}$, et si l'on suppose que les spins $K$ constituent une couche monoatomique sur la paroi, on trouve que le rapport du nombre de spins $S$ au nombre $N$ de spins $K$ est de $10^{-6}$. Par suite le nombre de spins $S$ qui, à un instant donné, interagissent avec un spin $K$ est de $10^{-12} N$. Le temps nécessaire pour que tout spin $K$ interagisse avec un spin $S$ est donc $\tau_{S} \times 10^{+12} \sim 100$ sec : c'est une limite inférieure du temps qui serait nécessaire pour polariser totalement les spins $K$ en l'absence de relaxation de ces derniers. Nous avons toutes les raisons de penser que le temps de relaxation des protons est très court devant 100 sec. et par suite qu'il n'existe pas d'orientation appréciable des protons sur la paroi.

Notons qu'en ce point, notre problème diffère de celui qui est traité explicitement dans la référence 3 : dans le cas présent les spins $K$ constituent le réseau ; nous faisons l'hypothèse que sa capacité calorifique est grande en sorte que l'interaction avec les spins $S$ ne modifie pas son état d'équilibre ; dans la référence 3 , le nombre de spins $S$ et de spins $K$ est comparable, il y a transfert de polarisation d'un système de spins à l'autre.

Plan du calcul. - Nous écrivons l'équation d'évolution de la matrice densité des atomes de rubidium sous l'effet de $\mathfrak{H}_{0}(S)$ et de $\mathfrak{H e}_{1}(t)$; nous en déduisons l'équation d'évolution des observables suivantes :

- la population d'un niveau hyperfin donné. Elle se déduit de la valeur moyenne de l'opérateur $F^{2}$ ou encore de l'opérateur S.I (étant donnée la relation $F^{2}=\frac{3}{4}+I(I+1)+2 S . I$.

- l'aimantation électronique longitudinale $<S_{z}>$ et transversale $<S^{+}>$;

- l'aimantation nucléaire longitudinale $\left\langle I_{z}\right\rangle$ et transversale $\left\langle I^{+}>\right.$.

Dans la première partie, nous envisageons le cas d'un champ $H_{0}$ faible et tel que le hamiltonien Zeeman $\mathfrak{H}_{z}=\omega_{S} S_{z}-\omega_{I} I_{z}$ soit petit devant le hamiltonien hyperfin al.S. $F$ reste un bon nombre quantique. Nous décrivons l'évolution de la matrice densité sous forme opératorielle et nous en déduisons l'évolution de chaque observable.

Dans la deuxième partie, nous donnons les résultats du calcul pour le cas inverse $a S . I \ll \mathcal{H}_{z}$.

Dans la troisième partie, nous calculons pour un champ $\mathrm{H}_{0}$ quelconque l'évolution de chaque élément de la matrice densité individuellement. Nous en déduisons l'équation d'évolution de $\left\langle S_{z}\right\rangle$ et $<S^{+}>$.

Dans la quatrième partie, nous donnons la généralisation de ces résultats au cas où l'interaction responsable de la relaxation n'agit sur la fonction d'onde de l'atome de Rb que par l'intermédiaire du spin électronique.

\section{PREMIÈRE PARTIE}

I. Introduction. - A) Hamiltonien D'interAction. - Nous prendrons pour hamiltonien d'interaction $\mathfrak{H}_{1}(t)$ entre les moments magnétiques $\mu_{S}$ et $\mu_{K}$ l'expression [5]:

$$
\begin{aligned}
\mathfrak{H}_{1}=-\frac{8 \pi}{3} \hbar_{\gamma_{S}} \gamma_{K K} \delta \delta_{r_{S K} \mid}^{3} S . K \\
+\frac{\hbar \gamma_{S K} \gamma}{r_{S K}^{3}}\left[S . K-3 \frac{\left(S . r_{S K}\right)\left(K . r_{S K}\right)}{r_{S K}^{2}}\right]
\end{aligned}
$$


$\mathfrak{H e}_{1}$ est fonction aléatoire du temps par l'intermédiaire des coordonnées de position relative de $\boldsymbol{\mu}_{S}$ et $\boldsymbol{\mu}_{K}: r_{S K}, \theta, \varphi$ (fig. 1).

Les deux termes de $\mathfrak{H}_{1}$ correspondent respectivement à l'interaction de contact (partie scalaire) et à l'interaction dipole-dipole (partie tensorielle).

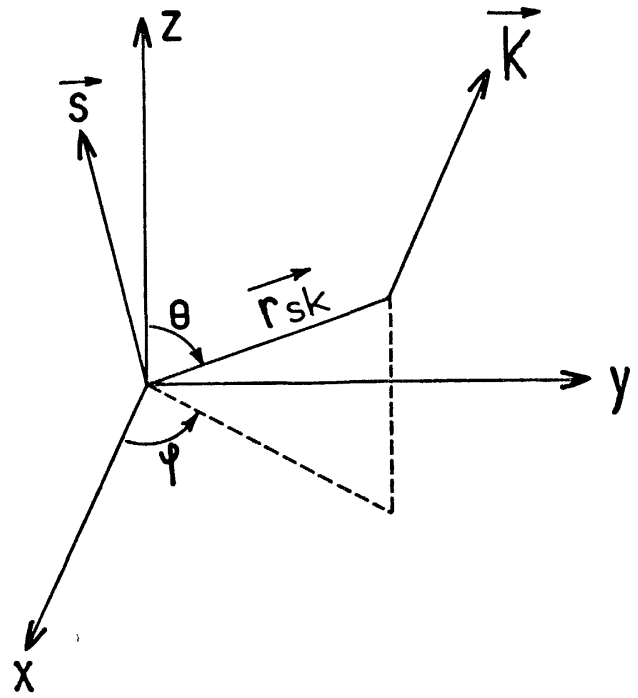

FIG. 1.

Suivant la méthode habituelle, nous décomposons $\mathcal{H}_{1}$ en opérateurs $A^{\text {lm }}$ dont la caractéristique est d'induire des transitions à $m$ quanta

$$
\left(\Delta\left(m_{S}+m_{K}\right)=m\right)
$$

entre 2 états propres de $\mathscr{H}_{0}$. Nous écrirons :

$$
\mathscr{K}_{1}(t)=\sum_{l m} a F^{l m}(t) A^{l m},
$$

avec les valeurs $l=0$ et $l=2$ pour les parties scalaires et tensorielles de l'interaction. Les notations sont les suivantes:

$$
a_{0}=\gamma_{S} \gamma_{K} \hbar 8 \pi / 3, \quad a_{2}=\gamma_{S} \gamma_{K} \hbar \sqrt{\pi / 5 .}
$$

Les grandeurs $F^{l m}(t)$ sont, par l'intermédiaire de $r, \theta, \varphi$, des fonctions aléatoires du temps de la forme :

$$
F^{l m}=f_{l}(r) Y^{l m}(\theta, \varphi) .
$$

$Y^{l m}(\theta, \varphi)$ sont les harmoniques sphériques d'ordre $l$ et de rang $m$

$$
f_{0}(r)=\delta_{\left|r_{S K}\right|}^{3}, \quad f_{2}(r)=1 / r^{3} .
$$

Nous avons choisi la phase des harmoniques sphériques de telle sorte que :

$$
F l m=F l-m * .
$$

Le moyennage par rapport à l'ensemble des spins revient à faire une moyenne par rapport à $\theta$, $\varphi$ et $r$. Comme toutes les valeurs de $\theta$ et $\varphi$ sont également probables, nous pourrons utiliser les relations d'orthogonalité des harmoniques sphériques :

$$
\int Y^{l m} Y^{l^{\prime} m^{\prime}} \mathrm{d} \Omega=\delta_{m,-m^{\prime}} \delta_{l \cdot l^{\prime}} .
$$

Les $A^{l m}$ sont des opérateurs qui agissent sur les composantes des spins $S$ et $K \quad\left(S^{(0)}=S_{z}\right.$

$$
S^{( \pm 1)}=\frac{1}{\sqrt{2}}\left(S_{x} \pm i S_{y}\right)
$$

et les quantités analogues pour $K$ ).

Leur expression générale est

$$
\begin{aligned}
& A^{l m}=\sum_{r} \alpha_{r}^{l m} S(r) K^{(m-r)} \\
& \left\{\begin{array}{l}
A^{00}=\sum_{r} S^{(r)} K^{(-r)} \\
A^{20}=-4 S^{(0)} K^{(0)}+2 S^{(-1)} K^{(1)}+2 S^{(1)} K^{(-1)} \\
A^{21}=-2 \sqrt{3} S^{(1)} K^{(0)}-2, \overline{3} S^{(0)} K^{(1)} \\
A^{2-1}=-2 \sqrt{3} S^{(-1)} K^{(0)}-2 \sqrt{3} S^{(0)} K^{(-1)} \\
A^{22}=-2 \sqrt{6} S^{(1)} K^{(1)} \\
A^{2-2}=-2 \sqrt{6} S^{(-1)} K^{(-1)}
\end{array}\right.
\end{aligned}
$$

On a les relations

$$
A^{l m}=A^{l-m^{\dagger}} \quad \text { ou } \quad \alpha_{r}^{l m}=\alpha_{r}^{l-m} .
$$

B) Hamiltonien statique. - Nous prendrons pour hamiltonien statique agissant sur les atomes et les spins $K: \mathfrak{H}_{0}(S)-\omega_{K} K_{z}=\mathfrak{H}_{0} ; \mathfrak{H}_{0}(S)$ est le hamiltonien défini par l'équation 1 .

En champ magnétique faible, chaque niveau hyperfin $F$ se décompose en $2 F+1$ sous-niveaux dont la distance est $\omega_{F}=g_{F}^{\prime} \omega_{S}, \omega_{S}$ étant la pulsation de Larmor du spin $S$ isolé et $g_{F}^{\prime}$ la quantité $g^{\prime}{ }^{\prime}=\frac{F(F+1)+S(S+1)-I(I+1)}{2 F(F+1)_{1}}$. Notons que

$$
g_{F_{+}^{\prime}}^{\prime}=-g_{F_{-}^{\prime}}^{\prime}=\frac{1}{2 I+1} .
$$

Les facteurs de Landé $g_{F}=2 g^{\prime}{ }_{F}$ des 2 niveaux $F_{+}$et $F_{-}$sont opposés. Nous supposons dans cette partie que $\omega_{F} / \Delta W \ll 1$ et que $F$ est un bon nombre quantique.

Introduisons les opérateurs de projection sur les états $F_{+}$et $F_{-}$respectivement :

$$
\begin{aligned}
P_{F+} & =\frac{2 S . I+I+1}{2 I+1} \quad P_{F_{-}}=-\frac{2 S . I-I}{2 I+1} \\
& <F^{\prime} m_{F}\left|P_{F \pm}\right| F m_{F}>=\delta_{F, F^{\prime}} \delta_{F, F \pm} .
\end{aligned}
$$

A l'aide de ces opérateurs, le hamiltonien $\mathscr{H}_{0}$ s'écrit : $\left(\frac{\omega_{K}}{\omega_{S}} \simeq 10^{-3} \ll 1\right)$

$$
\mathscr{H}_{0}=a \mathrm{~S} . I+\sum_{F_{+},{ }^{F}-} P_{F} g_{F}^{\prime} \omega_{S} F_{z}-\omega_{K} K_{z} .
$$

II. Équation d'évolution de la matrice densité. - Nous appelons $\sigma(S, K)$ la matrice densité de 
l'ensemble des atomes (de spin électronique $S$ ) et des spins $K$ de la paroi.

En représentation d'interaction :

$$
\sigma^{*}(S, K)=\mathrm{e}^{i \mathcal{H}_{0} t} \sigma(S, K) \mathrm{e}-i \mathcal{H}_{0} t
$$

$\mathscr{H}_{1}^{*}(t)=\mathrm{e}^{i \mathscr{H}_{0} t} \mathfrak{H}_{1}(t) \mathrm{e}-i \mathfrak{H}_{0} t$

$$
=\sum_{l m} a_{l} F l m(t) \alpha_{r}^{l m} \mathrm{e}^{i \mathcal{H}_{0} t} S(r) K^{(m-r)} \mathrm{e}-i \mathcal{H}_{0} t .
$$

Les opérateurs $S^{(r)}$ ne commutent pas avec le terme alS. Nous écrivons :

$$
S(r)=\sum_{p} S_{p}^{(r)} \quad p=0 \quad \text { ou } \quad \pm 1
$$

Par définition l'opérateur $S_{p}^{(r)}$ agissant sur un état $\mid F, m_{F}>$ augmente $F$ de $p$ unités : il n'a donc d'élément de matrice qu'entre l'état $\mid F, m_{F}>$ et l'état $\mid F+p, m_{F}+r>$.
La différence d'énergie au cours de la transition est :

$$
\begin{gathered}
p\left[\Delta W+\left|g_{F}^{\prime}\right| \omega_{S}\left(2 m_{F}+r\right)\right] \text { pour } p \neq 0 \\
\text { et } \quad r g_{F}^{\prime} \omega_{S} \text { pour } p=0 .
\end{gathered}
$$

Nous en déduisons

$\mathfrak{H}_{1}^{*}(t)=$

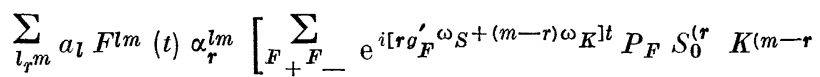

$\left.\left.+\sum_{p= \pm 1} \mathrm{e}^{i\left\{p\left[\Delta W+\left|g_{F}^{\prime}\right|{ }^{\omega} S^{(2 m} F+r\right)\right]+(m-r) \omega} K\right\} t S_{p}^{(r)} K^{(m-r)}\right]$

L'équation d'évolution de $\sigma^{*}(S, K)$ en représentation d'interaction est donnée dans la référence (3) : $\frac{\mathrm{d} \sigma_{(S, K)}^{*}}{\mathrm{~d} t}=-\int_{0}^{\infty} \mathrm{d} \tau \overline{\left[\mathcal{H}_{1}^{*}(t),\left[\mathcal{H}_{1}^{*}(t-\tau), \sigma_{(S, K)}^{*}(t)\right]\right.}$,

soit, dans le cas présent

$$
\begin{aligned}
& \frac{\mathrm{d} \sigma_{(S, K)}^{*}}{\mathrm{~d} t}= \\
& -\int_{0}^{\infty} \mathrm{d} \tau \overline{\left[\sum_{r}^{l_{m}} a_{l} F l m(t) \alpha_{r}^{l m} \mathrm{e}^{i \mathcal{H}_{0} t} S^{(r)} K^{(m-r)} \mathrm{e}-i \mathcal{H}_{0} t,\left[\sum_{l^{\prime} m^{\prime}} a_{l^{\prime}} F^{\prime} m^{\prime}(t-\tau) \alpha_{r^{\prime}}^{l^{\prime} m^{\prime}} \mathrm{e}^{i \mathcal{H}_{0}(t-\tau) S\left(r^{\prime}\right)} K^{\left(m^{\prime}-r^{\prime}\right.} \mathrm{e}-i_{0}(t-\tau), \sigma_{S) K)}^{*}(t)\right]\right]} .
\end{aligned}
$$

A partir de cette équation, le calcul s'effectue dans l'ordre suivant : Nous effectuons la moyenne par rapport aux coordonnées de position relative des spins $S$ et $K(\S 1)$, puis la moyenne par rapport aux orientation des spins $K(\$ 2)$, enfin nous simplifions l'équation d'évolution par l'approximation séculaire (§ 3). Le résultat du calcul est exprimé par les équations 31,32 et 33 ; elles permettent de calculer l'évolution d'une observable comme nous l'indiquons dans III.

$1^{\circ}$ Comme il résulte des équations (7) et (2) nous avons :

$$
\begin{aligned}
& \overline{F l m(t) F l^{\prime} m^{\prime}(t-\tau)}=\frac{\tau_{S}}{\tau_{S}+\tau_{V}} \widetilde{F}\left(m(t) F l^{\prime}, m^{\prime}(t) \mathrm{e}^{-\tau / \tau} c\right. \\
& =\frac{\tau_{S}}{\tau_{S}+\tau_{V}} \widetilde{f_{l}\left(r_{S K}(t)\right)} \tilde{f}_{l^{\prime}}\left(r_{S K}(t)\right) \\
& \widetilde{Y} l m\left(\theta_{(t)} \varphi(t t)\right) \tilde{Y^{\prime} m^{\prime}\left(\theta_{(t)} \varphi(t)\right)} e^{-\tau^{\prime} \tau_{c}}
\end{aligned}
$$

$f_{l}\left(r_{S K}\right)$ étant homogène à l'inverse du cube d'une longueur, nous poserons :

$$
\widetilde{\left|f_{l}\left(r_{S K}(t)\right)\right|^{2}}=1 / R_{l}^{6},
$$

la moyenne étant prise sur l'ensemble des atomes $q u i, \dot{a}$ un instant $t$ donné, sont sur la paroi.
Utilisant les relations d'orthogonalité des $Y^{l m}$, il vient alors :

$$
F l m(t) F l^{\prime} m^{\prime}(t-\tau)=\frac{\tau_{S}}{\tau_{S}+\tau_{V}} \frac{1}{R_{l}^{6}} \mathrm{e}^{-\tau / \tau_{\mathrm{c}}} \delta_{\ell, l^{\prime}} \delta_{m,-m^{\prime}}
$$

$2^{\circ}$ Les observables $Q$ qui nous intéressent ne concernant que les atomes :

avec

$$
<Q>=\operatorname{Tr}\{\sigma(S, K) Q\}=\operatorname{Tr}\{\sigma Q\},
$$

$$
\sigma=\operatorname{Tr}_{K} \sigma(S, K) \text {. }
$$

Nous chercherons donc directement à déterminer l'évolution de $\sigma^{*}(t)$ : dans l'équation (21), nous prendrons la trace de chaque membre relativement aux spins $K$. Nous voyons apparaitre des expressions de la forme :

$$
\operatorname{Tr}_{K} \overline{\left\{K^{(m-r)} K^{\left(-m-r^{\prime}\right)} \sigma(S, K)\right\}} \text {. }
$$

Comme nous l'avons précisé dans l'introduction, nous supposons que les spins $K$ demeurent à l'équilibre thermique malgré leur interaction avec les spins $S$; la matrice densité des spins $K$ reste proportionnelle à la matrice unité et la valeur de l'expression ci-dessus se déduit de l'équation (3), c'est : $\frac{K(K+1)}{3} \delta_{r_{r}-r^{\prime}} \sigma$.

$$
\begin{aligned}
& \operatorname { T r } _ { K } \longdiv { \mathrm { e } ^ { i \omega _ { K } K ^ { ( 0 ) } t } K ^ { ( m - r ) } \mathrm { e } ^ { - i \omega _ { K } K ^ { ( 0 ) } t } \sigma _ { K } ^ { * } ( t ) \mathrm { e } ^ { i \omega _ { K } K ^ { ( 0 ) } ( t - \tau ) } K ^ { ( m ^ { \prime } - r ^ { \prime } ) } \mathrm { e } ^ { - i \omega _ { K } K ^ { ( 0 ) } ( t - \tau ) } } \\
& =\operatorname{Tr}_{K} \overline{\left.\mathrm{e}^{i \omega_{K}}{ }^{(0)} t K^{(m-r} \mathrm{e}^{-i \omega_{K} K^{(0)} \tau} K^{\left(m^{\prime}-r^{\prime}\right)} \mathrm{e}^{-i \omega_{K} K^{(0)}(t-\tau)} \sigma_{(S, K)}^{*}(t)\right\}}=\delta_{r,-r^{*}} \delta_{m,-m^{\prime}} \mathrm{e}^{i(m-r) \omega_{K} \tau} \frac{K(K+1)}{3} \sigma^{*} .
\end{aligned}
$$


En effectuant les moyennes indiquées dans les paragraphes 1 et 2, l'équation (21) devient :

$$
\begin{aligned}
& \frac{\mathrm{d} \sigma^{*}}{\mathrm{~d} t}=-\sum_{r m} \frac{\left|a_{l}\right|^{2}}{R_{l}^{6}}\left|\alpha_{r}^{7 m}\right|^{2} \frac{\tau_{S}}{\tau_{S}+\tau V} \frac{K(K+1)}{3} \\
& \int_{0}^{\infty} \mathrm{d} \tau \mathrm{e}^{i(m-r) \omega_{K} \tau-\tau / \tau_{\mathrm{c}} \times} \\
& \quad\left[\mathrm{e}^{i \mathcal{H}_{0} t} S(r) \mathrm{e}-i \mathcal{H}_{0} t\left[\mathrm{e}^{i \mathcal{H}_{\theta}(i-\tau)} S(-r) \mathrm{e}^{\left.\left.i \mathcal{H}_{0}(t-\tau), \sigma_{(t)}^{*}\right]\right]}\right.\right.
\end{aligned}
$$

Nous poserons

$$
k_{l}=\frac{\left|a_{l}\right|^{2}}{2 R_{l}^{6}} \frac{\tau_{S}}{\tau_{S}+\tau_{V}} \frac{K(K+1)}{3} .
$$

$3^{\circ}$ Nous étudions maintenant le double commutateur qui figure dans l'équation (23) dans le but de simplifier cette équation par "l'approximation séculaire ».

$$
\begin{aligned}
& \left.\left[\begin{array}{l}
\mathrm{e}^{i \mathcal{H}_{0} t} S^{(r)} \mathrm{e}^{-i \mathcal{H}_{0} t},\left[\mathrm{e}^{i \mathcal{H}_{0}(t-\tau)} S^{(-r)} \mathrm{e}^{-i \mathcal{H}_{0}(t-\tau)} \sigma_{(t)}^{*}\right]= \\
\sum_{p p^{\prime}}\left[\mathrm{e}^{i \mathcal{H}_{0} t} S_{p}^{(r)} \mathrm{e}^{-i \mathcal{H}_{0} t},\left[\mathrm{e}^{i \mathcal{H}_{0}(t-\tau)} S_{p^{\prime}}^{(-r)} \mathrm{e}^{-i \mathcal{H}_{0}(t-\tau),},{ }_{(t)}^{*}\right.\right.
\end{array}\right]\right] .
\end{aligned}
$$

Dans cette somme certains termes dits séculaires sont indépendants de $t$, d'autres oscillent en fonction du temps. Si l'on ne tient compte que des termes séculaires l'équation d'évolution de $\sigma^{*}(t)$ représente un système différentiel linéaire à coefficients constants par rapport aux variables $\sigma_{F m}^{* F m_{\mathrm{F}}}$. Nous pouvons trouver sa solution : comme nous le verrons par la suite un élément de matrice $\sigma_{F m}^{*} \underset{F}{F}$ évolue avec plusieurs constantes de temps dont les valeurs restent toujours assez voisines de celles $\left(T_{1} \ldots\right)$ qui interviennent dans l'évolution de $\left\langle S_{z}\right\rangle$. On démontre [7] que l'existence des termes non séculaires oscillant à la fréquence $\Delta W / 2 \pi$ ou $\left|g^{\prime}{ }_{F}\right| \omega_{S} / 2 \pi$ apporte respectivement une correction d'ordre $1 / \Delta W T_{1}$ ou $1 /\left|g^{\prime}{ }_{F}\right| \omega_{S} T_{1}$ à la solution précédente de $\sigma^{*}(t)$. Pour que cette correction soit négligeable, il suffit done que la condition $\left|g^{\prime}{ }_{F}\right| \omega_{S} T_{1} \gg 1$ soit réalisée. Expérimentalement, nous avons mesuré des temps de relaxation $T_{1} \geqslant 0,1 \mathrm{~s}$. Il suffit done que le champ statique soit grand devant $10^{-6}$ gauss. Cette condition est toujours très largement satisfaite dans nos expériences.

Par suite, nous ne garderons dans le second membre de l'équation (23) que les termes séculaires. Nous cherchons à éliminer d'abord les termes oscillant à la fréquence $\Delta W$. Pour cela, on doit limiter la somme sur les indices $p$ et $p^{\prime}$ aux termes pour lesquels $p^{\prime}=-p$ : les opérateurs $S_{p}^{(r)}$ et $S_{p^{\prime}}^{\left(-^{r}\right)}$ produisent en effet des transitions entre niveaux dont la différence d'énergie (à $\omega_{F}$ près, $\omega_{F} \ll \Delta W$ ) est $p \Delta W$ et $p^{\prime} \Delta W$. Une conséquence de l'équation $p+p^{\prime}=0$ est que les termes diagonaux en $F$ de la matrice densité sont uniquement couplés aux autres termes diagonaux sous l'effet de la relaxa- tion et que les cohérences hyperfines sont uniquement couplées aux autres cohérences hyperfines. A l'instant initial la matrice $\sigma^{*}(t)$ est diagonale en $F$ et nous voyons donc que cette propriété subsiste sous l'effet de la relaxation. Nous pouvons décomposer la matrice $\sigma^{*}$ en 2 sous-matrices correspondant chacune àun niveau hyperfin :

$$
\begin{aligned}
& \sigma^{*}=\sigma_{F_{+}}^{*}+\sigma_{F_{-}}^{*} \\
& \sigma_{F_{+}}^{*}=P_{F_{+}} \sigma^{*} P_{F_{+}} \\
& \sigma_{F_{-}}^{*}=P_{F_{-}} \sigma^{*} P_{F_{-}} .
\end{aligned}
$$

Nous étuḍierons séparément l'équation d'évolution de chacune de ces sous-matrices. Posons

$$
S_{p}^{(r) *}(t)=\mathrm{e}^{i \mathcal{H}_{0} t} S_{p}^{(r)} \mathrm{e}-i \mathcal{H}_{0} t .
$$

Nous déduisons de l'équation (23).

$$
\begin{aligned}
& \frac{\mathrm{d}}{\mathrm{d} t} \sigma_{F_{ \pm}}^{*}=-\left.\left.\sum_{l_{r} m} 2 k_{l}\right|^{\alpha}{ }_{r}^{l m}\right|^{2} \int_{0}^{\infty} \mathrm{d} \tau \mathrm{e}^{i(m-r) \omega} K^{\tau} \mathrm{e}^{-\tau / \tau_{\mathbf{c}}} \times
\end{aligned}
$$

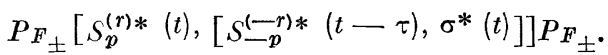

A ce stade, il nous reste à éliminer les termes non séculaires à la fréquence $\omega_{S}$. Il est nécessaire pour cela de développer les doubles commutateurs de l'équation (25) et d'examiner séparément l'évolution dans le temps des différents termes obtenus. L'algèbre correspondante est assez lourde et nous ne la reproduisons pas. Nous donnerons le résultat algébrique et son interprétation physique :

Nous définissons la matrice $\overline{\sigma_{F \pm}^{*}(t)}$ comme étant la matrice qui a les mêmes éléments diagonaux que $\sigma_{F \pm}^{*}(t)$ mais qui a tous ses éléments non diagonaux nuls :

$$
\begin{aligned}
& <F m_{F}\left|\overline{\sigma_{F_{ \pm}}^{*}(t)}\right| F m_{F}^{\prime}> \\
& \quad=<F m_{F}\left|P_{F_{ \pm}} \sigma^{*}(t) P_{F_{ \pm}}\right| F m_{F}^{\prime}>\delta_{m_{F} m_{F}^{\prime}} .
\end{aligned}
$$

Nous négligeons $\omega_{K} / \Delta W$ et $\omega_{F} / \Delta W$ devant l'unité. On trouve alors l'équation d'évolution suivante :

$$
\begin{aligned}
& \frac{\mathrm{d}}{\mathrm{d} t} \sigma_{F_{+}}^{*}=-\sum_{r m} k_{l}\left|\alpha_{r}^{l m}\right|^{2}\left\{\left[S_{0}^{(r)},\left[S_{0}^{(-r)}, \sigma_{F_{+}}^{*}(t)\right]\right]\right.
\end{aligned}
$$

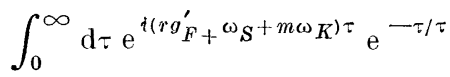

$$
\begin{aligned}
& +\sum_{p= \pm 1} P_{F}+\left[S_{p}^{(r)},\left[S_{-p}^{(-r)}, \sigma_{F_{+}}^{*}(t)\right]\right] P_{F+} \\
& \int_{0}^{\infty} \mathrm{d} \tau \mathrm{e}^{i p \Delta W^{\prime} \tau} \mathrm{e}^{-\tau / \tau} \\
& +\sum_{p= \pm 1} P_{F+[}\left[S_{p}^{(r)},\left[S_{-p}^{(-r)}, \overline{\sigma_{F_{-}}^{*}(t)}\right]\right]^{P_{F+}} \\
& \left.\int_{0}^{\infty} \mathrm{d} \tau \mathrm{e}^{i p \Delta \omega \tau} \mathrm{e}^{\tau / \tau} \mathrm{c}\right\}
\end{aligned}
$$


(l'équation d'évolution de $\sigma_{F-}^{*}(t)$ s'obtient en échangeant $F_{+}$et $F_{-}$). Dans le référentiel du laboratoire

$$
\sigma_{F m_{F}}^{F m_{F}}(t)=\sigma_{F m_{F}^{*} F m_{F}^{\prime}}(t) \mathrm{e}^{-i \omega_{F m} m_{F} F m_{F}^{\prime} t} ;
$$

cette cohérence évolue à la fréquence $\omega_{F m_{F}} F m^{\prime}$ (distance des niveaux $F m_{F}, F m_{F}^{\prime}$ ). Le résultat de l'approximation séculaire est que les équations de la relaxation ne couplent entre eux que les éléments de la matrice densité qui ont même fréquence pro-

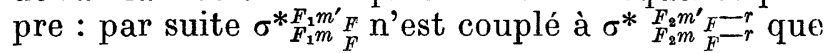
dans l'un ou l'autre des 2 cas suivants :

$1^{\mathrm{er}}$ cas :

$$
\omega_{F_{1} m_{F} F_{1} m_{F}^{\prime}}=\omega_{F_{\mathbf{2}} m_{F^{-}}-r F_{2} m_{F}^{\prime}-r}=0 .
$$

Ceci impose $m_{F}=m_{F}^{\prime}\left(F_{1}\right.$ et $F_{2}$ pouvant être quelconques). Ce cas concerne les populations : les populations $\sigma_{F m_{F}^{F}}^{F m_{F}}$ sont couplées entre elles mais elles ne sont pas couplées aux cohérences.

L'étude théorique et expérimentale de l'évolution des populations (calcul et mesure de $T_{1}$ ) peut donc se faire sans que l'on ait à se préoccuper de l'existence d'une cohérence Zeeman dans l'état initial.

$2^{\mathrm{e}}$ cas :

$$
\omega_{F_{1} m_{F}, F_{2} m_{F}^{\prime}}=\omega_{F_{\mathbf{8}} m_{F^{F}} \longrightarrow r, F_{2} m_{F}^{\prime}-r} \quad \operatorname{avec} m_{F} \neq m_{F}^{\prime} .(f i g .2 a) .
$$

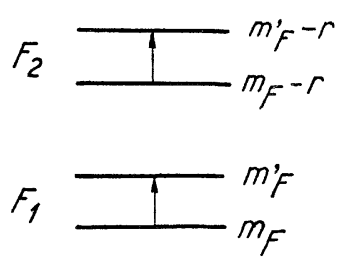

$a$
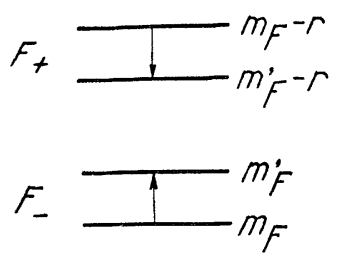

$b$
FIG. 2.

Il est clair que si les facteurs de Landé de $F_{1}$ et de $F_{2}$ sont différents, l'égalité précédente n'est possible que si $F_{1}=F_{2}$. C'est en particulier ce qui se passe pour les sous-niveaux $F_{+}$et $F_{-}$de l'état fondamental des alcalins : il ne peut y avoir de transfert de cohérence d'un niveau hyperfin à l'autre parce que les fréquences de Larmor de $F_{+}$et $F_{-}$sont de signes opposés (fig. $2 b$ ). On peut montrer que la condition réelle de couplage de deux cohérences est en fait moins restrictive que l'égalité précédente. Il suffit, en fait, que

$$
\left(\omega_{F m_{F}, F m_{F}}-\omega_{\left.F m_{F}-r, F m_{F}^{\prime}-r\right)} T_{2} \ll 1 .\right.
$$

Deux cohérences Zeeman $\left(\sigma_{F m}^{F m_{F}} \underset{F}{ }\right.$ et $\left.\sigma_{F m_{F}^{F}}^{F m_{F}^{\prime}-r}\right)$ sont couplées à l'intérieur d'un niveau $F$ si les résonances correspondantes sont confondues à la largeur de relaxation près.

Nous avons conduit le calcul et écrit l'équation de relaxation (29) dans le cas où cette dernière condition est remplie, c'est-à-dire puisque $r= \pm 1$, dans le cas où la distance de 2 composantes Zeeman consécutives est petite par rapport à la largeur de relaxation de l'une d'elle. Ceci entraîne pour la valeur du champ magnétique la condition : $H_{0}<\bar{H}_{0}, \bar{H}_{0}$ étant la valeur pour laquelle : $\left(\omega_{F m_{F}}, \quad F_{F m^{\prime}}-\omega_{F m_{F}-1}, \quad F m_{F}^{\prime}-1\right) T_{2}=1$. Nous limitons donc dans la partie actuelle de notre travail-l'étude de l'aimantation transversale à ce cas particulier d'un champ très faible (l'étude pour un champ $H_{0}$ quelconque étant faite dans la troisième partie). Il est à noter que cette restriction ne joue pas en ce qui concerne les populations $\sigma_{F m_{F}^{F}}^{F m_{F}}$ dont nous avons vu qu'elles ne sont pas couplées aux cohérences. Comme $\left\langle S_{\mathrm{z}}\right\rangle$ et $\left\langle I_{\mathrm{z}}\right\rangle$, aimantations longitudinales, sont des combinaisons linéaires des populations, on voit que la condition de validité de notre étude relative à $\left\langle S_{z}\right\rangle$ et $\left\langle I_{\mathrm{z}}>\right.$ n'est pas, dans ce chapitre, $H_{\mathbf{0}}<\vec{H}_{0}$, mais simplement que $H_{0}$ soit assez faible pour que $F$ soit un bon nombre quantique.

Pour achever la transformation de l'équation 29, notons que dans son second membre figurent 3 intégrales du même type :

$$
\int_{0}^{\infty} \mathrm{d} \tau \mathrm{e}^{i \Omega \tau} \mathrm{e}^{-\tau / \tau_{\mathrm{c}}}=\frac{1}{2} J(\Omega)-i k(\Omega),
$$

$J(\Omega)$ est la transformée de Fourier pour la fréquence $\Omega / 2 \pi$ de la fonction de corrélation de la perturbation :

$$
J(\Omega)=2 \tau_{\mathrm{c}} /\left(1+\Omega^{2} \tau_{\mathrm{c}}^{2}\right) .
$$

(On peut montrer que le terme imaginaire $i k(\Omega)$ produit un déplacement d'énergie inférieur à la largeur des niveaux du système provoquée par la relaxation. Nous ne cherchons pas à tenir compte de cet effet).

Les valeurs de $\Omega$ qui interviennent sont finalement $\Delta W$ et $r g_{F}^{\prime} \omega_{S}+m \omega_{K}$. L'équation (29) peut donc être écrite :

$\frac{\mathrm{d}}{\mathrm{d} t} \sigma_{\vec{F}+}^{*}=$

$$
\begin{aligned}
& -\sum_{l_{r} m} k_{l}\left|\alpha_{r}^{l m}\right|^{2}\left\{\left[S_{0}^{(r)},\left[S_{0}^{(-r) 6} \sigma_{F_{+}}^{*}(t)\right]\right] J\left(r g_{F}^{\prime} \omega_{S}+m \omega_{K}\right)\right. \\
& +\sum_{p= \pm 1} P_{F+}\left[S_{p}^{(r)},\left[S_{-p}^{(-r)}, \sigma_{F_{+}}^{*}(t)\right]\right] P_{F_{+}} J(\Delta W) \\
& +\sum_{p= \pm 1} P_{F_{+}}\left[\stackrel{\left.\left.S_{p}^{(r)},\left[S_{-p}^{(-r)}, \overline{\sigma_{F-}^{*}(t)}\right]\right] P_{F_{+}} J\left(\Delta W^{*}\right)\right\}}{ }\right.
\end{aligned}
$$

L'équation d'évolution de $\sigma_{F-}^{*}(t)$ s'en déduit en échangeant $F_{+}$et $F_{-}$.

$\mathrm{d} \sigma_{F}^{*} / \mathrm{d} t$ dépend linéairement des fonctions $J\left(r g_{F}^{\prime} \omega_{S}+m \omega_{R}\right)$ et $J(\Delta W)$; nous ferons un changement de variables et nous écrirons $\mathrm{d} \sigma_{F}^{*} / \mathrm{d} t$ 
en fonction de $\left(J\left(r g_{F}^{\prime} \omega_{S}+m \omega_{K}\right)-J(\Delta W)\right)$ et $J(\Delta W)$. Dans ces conditions l'interprétation physique de chaque terme devient simple : ils sont nuls respectivement dans l'un ou l'autre des deux cas extrêmes suivants :

$$
\begin{gathered}
J(\Delta W)=J\left(\omega_{F}\right)=J\left(\omega_{K}\right)=J(0), \\
{\left[\frac{\mathrm{d} \sigma_{F_{+}}^{*}}{\mathrm{~d} t}\right]_{1}=-\sum_{l_{r} m} k_{l}\left|\alpha_{r}^{l m}\right|^{2}\left[S_{0}^{(r)},\left[S_{0}^{(-r)}, \sigma_{F_{+}}^{*}(t)\right]\right]\left(J\left(r g_{F}+\omega_{S}+m \omega_{K}\right)-J(\Delta W)\right) .} \\
{\left[\frac{\mathrm{d} \sigma_{F_{+}}^{*}}{\mathrm{~d} t}\right]_{2}=-\sum_{l_{r p} m^{m}} k_{l}\left|\alpha_{r}^{l m}\right|^{2}\left\{P_{F^{+}}\left[S_{p}^{(r)},\left[S_{-p}^{(-r)}, \sigma_{F_{+}}^{*}(t)\right]\right] P_{F^{+}}+P_{F_{+}}\left[S_{p}^{(r)},\left[S_{-p}^{(-r)}, \overline{\sigma_{F_{-}}^{*}(t)}\right]\right] P_{F+}\right\} J(\Delta W) .}
\end{gathered}
$$

cas du rétrécissement extrême $\tau_{\mathrm{c}} \ll 1 / \Delta W$, et

$$
J \Delta(\boldsymbol{W})=0,
$$

cas où $\tau_{\mathrm{c}} \gg 1 / \Delta W$.

Nous écrirons donc $: \mathrm{d} \sigma^{*} / \mathrm{d} t=\left(\mathrm{d} \sigma^{*} / \mathrm{d} t\right)_{1}+\left(\mathrm{d} \sigma^{*} / \mathrm{d} t\right)_{2}$.

avec $p=0, \pm 1$.

III. Équation d'évolution des observables. Nous devons calculer $\left\langle Q>{ }^{*}=\operatorname{Tr}\left\{\sigma^{*} Q\right\}, Q\right.$ étant l'opérateur représentant l'observable Q. Nous donnons les résultats du calcul successivement dans le cas où $J(\Delta W)=0$ et dans le cas du rétrécissement extrême. L'équation d'évolution dans le cas général ( $\tau_{c}$ quelconque) se déduit immédiatement de l'étude de ces 2 cas particuliers (notés par les indices 1 et 2), comme nous l'avons vu à la fin du paragraphe précédent

$$
\frac{\mathrm{d}<Q>*}{\mathrm{~d} t}=\left[\frac{\mathrm{d}<Q>*}{\mathrm{~d} t}\right]_{1}+\left[\frac{\mathrm{d}<Q>*}{\mathrm{~d} t}\right]_{2},
$$

avec

$$
\left[\frac{\mathrm{d}<Q>>^{*}}{\mathrm{~d} t}\right]_{q}=\operatorname{Tr}\left\{Q\left[\frac{\mathrm{d} \sigma_{F}^{*}}{\mathrm{~d} t}\right]_{Q}\right\}+\operatorname{Tr}\left\{Q\left[\frac{\mathrm{d} \sigma_{F}^{*}}{\mathrm{~d} t}\right]_{q}\right\},
$$

$q$ représentant l'un des indices 1 ou 2 .

Nous avons groupé en appendice des remarques générales qui permettent de simplifier l'algèbre et des équations qui représentent les diverses étapes de ce calcul pour les différents cas. On aboutit aux résultats suivants :

A) Calcul de $\left[\frac{\mathrm{d}<Q>*}{\mathrm{~d} t}\right]_{1} \quad$ (C'est-A-dire AUSSI $\frac{\mathrm{d}\langle Q\rangle *}{\mathrm{~d} t}$ DANS LE CAS OU $J(\Delta W)=0$.)

On peut montrer que, plus généralement, les résultats de ce paragraphe sont valables dans le cas où $(2 I+1)^{2} J(\Delta W) \ll J\left(\omega_{F}\right)$.

$1^{\circ}$ Évolution de la population globale d'un niveau hyperfin donné : $Q=$ SI.

On trouve :

$$
\left[\frac{\mathrm{d}<\mathrm{s.I}>}{\mathrm{d} t}\right]_{1}=0 .
$$

Comme on pouvait s'y attendre le temps de relaxation de la population d'un niveau hyperfin est infiniment long si le spectre de Fourier de la perturbation ne contient pas la fréquence de transition hyperfine. $2^{\circ}$ Évolution des aimantations électronique (et nucléaire) longitudinales : $Q=S_{z}$ (et $Q=I_{z}$ ).

On trouve :

$$
\left[\frac{\mathrm{d}}{\mathrm{d} t}<S_{z}>\right]_{1}=-\sum_{l} 2 k_{l}\left|\alpha_{l}\right|^{2} \frac{J\left(\omega_{F}-J(\Delta W)\right.}{(2 I+1)^{2}}<S_{z}>.
$$

$\left|\alpha_{l}\right|^{2}$ est défini dans l'appendice :

$$
\left|\alpha_{l}\right|^{2}=\sum_{m}\left|\alpha_{r}^{l m}\right|^{2} \text { (indépendant de } r \text { ). }
$$

L'équation d'évolution que l'on obtient pour $\left\langle I_{z}\right\rangle$ se déduit de (36) en substituant dans les 2 membres $I_{z}$ à $S_{z}$. Dans l'hypothèse $(2 I+1)^{2} J(\Delta W) \ll J\left(\omega_{F^{\prime}}\right),<S_{z}>,\left(\right.$ et $\left.<I_{z}>\right)$ (relaxent avec une seule constante de temps $T$ et $T_{n}^{\prime}$ )

$$
\frac{1}{T_{\mathrm{e}}^{\prime}}=\frac{1}{T_{n}^{\prime}}=\sum_{l} 2 k_{l}\left|\alpha_{l}\right|^{2} \frac{J\left(\omega_{F}\right)}{(2 I+1)^{2}}=\frac{1}{T_{1}^{\prime}} .
$$

Nous verrons par la suite que ce cas est le seul où $\left\langle S_{z}\right\rangle$ relaxe exponentiellement. Expérimentalement, nous n'avons pas observé de déviations par rapport à une exponentielle unique pour la relaxation de ${ }^{87} \mathrm{Rb}$ sur des enduits hydrogénés. Cependant, comme nous l'expliquerons plus loin, ceci n'implique pas que nous nous trouvions en pratique rigoureusement dans le cas analysé ici.

Un calcul identique peut se faire dans le cas d'un spin $S$ isolé ; on trouve que $\left\langle S_{z}\right\rangle$ relaxe exponentiellement avec la constante de temps $T_{S_{1}}$.

$$
1 / T_{S_{1}}=\sum_{l} 2 k_{l}\left|\alpha_{l}\right|^{2} J\left(\omega_{S}\right) .
$$

(37 bis)

En comparant les expressions de $T_{e}^{\prime}$ et $T_{S_{1}}$, nous remarquons l'influence du spin nucléaire 1 : en champ faible, il donne une valeur du temps de relaxation de $\left\langle S_{z}\right\rangle$ qui est $(2 I+1)^{2}$ fois plus longue que celle du même spin $S$ isolé. On comprend qualitativement ce fait en remarquant qu'en présence du couplage aS.I la relaxation de $\left\langle S_{z}\right\rangle$ se fait "en cascade " par une suite de transitions d'un sous-niveau Zeeman à un autre, tandis qu'en l'absence de spin I une seule transition suffit à retourner l'orientation du spin électronique.

En champ faible, pour un enduit donné, on 
trouve, dans l'hypothèse $J(\Delta W)=0$, que les constantes de temps $T_{1}^{\prime}$ pour les deux isotopes du rubi$\operatorname{dium}^{85} \mathrm{Rb}(\operatorname{spin} I=5 / 2)$ et ${ }^{87} \mathrm{Rb}(\operatorname{spin} I=3 / 2)$ sont théoriquement dans le rapport 2,25 : expérimentalement, nous mesurons un rapport 2. Remarquons que cette propriété de l'interaction dipoledipole et de l'interaction de contact existe aussi pour toutes les interactions que l'on peut représenter par un hamiltonien $\mathfrak{J e}_{1}$ n'agissant que sur $S$ et ayant un $\tau_{\text {c }}$ tel que $(2 I+1)^{2} J(\Delta W) \ll J\left(\omega_{F}\right)$. Ce point sera démontré dans la $4^{\mathrm{e}}$ partie. Par suite, la connaissance de la valeur du rapport des $T_{1}$ des 2 isotopes du Rubidium ne permet pas de décider de la nature de l'interaction ; mais elle est compatible avec l'interaction décrite dans le prọsent travail. Si l'on admet le résultat expérimental que dans les cellules enduites de paraffines hydrogénées, l'interaction prédominante est celle de $S$ avec les protons de la paroi, la valeur expérimentale de ce rapport permet alors de comparer $\tau_{\mathrm{c}}$ à $1 / \Delta W$.

La variation de $1 / T_{\mathrm{e}}^{\prime}$ en fonction du champ magnétique (dans la zone où $\omega_{F} \ll \Delta W$ ) est en $J\left(\omega_{F}\right)=2 \tau_{c} /\left(1+\omega_{F}^{2} \tau_{c}^{2}\right)$. Elle peut être différente pour les deux interactions scalaires et tensorielle si leurs temps de corrélation sont différents.

$3^{\circ}$ Aimantation électronique (et nucléaire) transversale $Q=S^{(+\mathbf{1})}\left(\right.$ et $\left.Q=I^{(+1)}\right)$.

On trouve :

$$
\begin{aligned}
{\left[\frac{\mathrm{d}}{\mathrm{d} t}<P_{F}+S^{(+1)}>*\right] } & =-\sum_{l m} k_{l}\left\{\left|\alpha_{0}^{l m}\right|^{2} J\left(m \omega_{K}\right)\right. \\
+\left|\alpha_{1}^{l m}\right|^{2} J\left(\omega_{F}\right) & \left.-\left(\left|\alpha_{0}^{l m}\right|^{2}+\left|\alpha_{1}^{I m}\right|^{2}\right) J(\Delta W)\right\} \\
& \times \frac{1}{(2 I+1)^{2}}<P_{F} S(+1)>* .
\end{aligned}
$$

Les équations que l'on obtient pour l'évolution de $\left\langle P_{F-} S^{+1}\right\rangle^{*}$, de $\left\langle P_{F+} I^{(+1)}\right\rangle^{*}$ et de $\left\langle P_{F-} I^{(+1)}\right\rangle{ }^{*}$ sont identiques à (38), c'est-à-dire du type :

$$
\left[\frac{\mathrm{d}}{\mathrm{d} t}<Q>*\right]_{1}=-\frac{1}{T_{2}^{\prime}}<Q>*
$$

Remarquons que dans le référentiel du laboratoire les valeurs moyennes de $S^{(+1)}$ et $I^{(+1)}$ à l'intérieur des niveaux $F_{+}$et $F_{-}$précessent respectivement autour de $H_{0}$ à la vitesse angulaire $\omega_{\mathrm{s}} /(2 I+1)$ et $-\omega_{\mathrm{s}} /(2 I+1)$. Notre résultat signifie que l'amortissement de chacune de ces précessions se fait avec une seule constante de temps $\left(T_{2}^{\prime}\right)_{F+}$ et $\left(T_{2}^{\prime}\right)_{F-}$ et que de plus

$$
\left(T_{2}^{\prime}\right)_{F+}=\left(T_{2}^{\prime}\right)_{F-}=T_{2}^{\prime} .
$$

Dans la zone de champ magnétique où $J\left(\omega_{F}\right)=J(0)$ (toujours dans le cas

$$
(2 I+1)^{2} J(\Delta W) \ll J\left(\omega_{F}\right),
$$

nous obtenons le résultat remarquable :

$$
T_{2}^{\prime}=T_{1}^{\prime}
$$

les temps de relaxation longitudinal et transversal sont uniques et égaux. Nous reviendrons plus loin sur l'interprétation physique de ce résultat.

La variation de $1 / T_{2}^{\prime}$ en fonction du champ magnétique $\left(H_{0}<\overline{H_{0}}\right)$ est différente pour les interactions scalaire (39) et tensorielle (40).

$$
\begin{aligned}
& \frac{1}{T_{2}^{\prime}}=2 k_{0}\left|\alpha_{0}\right|^{2} \frac{J(0)+J\left(\omega_{F}\right)-2 J(\Delta W)}{2(2 I+1)^{2}} \\
& \frac{1}{T_{2}^{\prime}}=2 k_{2}\left|\alpha_{2}\right|^{2} \frac{2 J(0)+3 J\left(\omega_{K}\right)+5 J\left(\omega_{F}\right)-10 J(\Delta W)}{10(2 I+1)^{2}} .
\end{aligned}
$$

En pratique la valeur de $\tau_{\mathrm{c}}$ est trop courte pour qu'il soit possible d'observer cette différence : dans tous les champs magnétiques où nous opérons $J\left(\omega_{K}\right)=J(0)$.

B) Calcul de $\left[\frac{\mathrm{d}<Q>^{*}}{\mathrm{~d} t}\right]_{2}$ (C'EST-A-dire AUSSI $\frac{\mathrm{d}<Q\rangle^{*}}{\mathrm{~d} t}$ DANS LE CAS DU RÉTRÉCISSEMENT $\left.\operatorname{EXTREMME}) .-1^{0}\right) \quad Q=$ S.I.

On trouve

$\left[\frac{\mathrm{d}}{\mathrm{d} t}<\mathrm{s} . I>\right]_{2}=-\sum_{l} 2 k_{l}\left|\alpha_{l}\right|^{2} J(\Delta W)<s . l>$.

Les transitions entre niveaux hyperfins étant possibles dans le cas du rétrécissement extrême, les populations de $F_{+}$et $F$ - évoluent exponentiellement au cours du temps avec un temps de relaxation $T_{H}^{\prime \prime}$.

$$
\frac{1}{T_{H}^{\prime \prime \prime}}=\sum_{l} 2 k_{l}\left|\alpha_{l}\right|^{2} J(0) .
$$

$\left.2^{\circ}\right) \quad Q=S_{z}$ et $Q=I_{z}$.

$$
\begin{aligned}
{\left[\frac{\mathrm{d}}{\mathrm{d} t}<S_{z}>\right]_{2}=} & -\sum_{l} 2 k_{l}\left|\alpha_{l}\right|^{2} J(\Delta W) \\
& {\left[<S_{z}>-\frac{2}{(2 I+1)^{2}}<I_{z}>\right] . }
\end{aligned}
$$

$$
\left[\frac{\mathrm{d}}{\mathrm{d} l}<I_{z}>\right]_{2}=-\sum_{l} \cdot 2 k_{l}\left|x_{l}\right|^{2} J(\Delta W) \frac{2}{(2 I+1)^{2}}<I_{z}>.
$$

Ainsi $<I_{z}>$ évolue exponentiellement au cours du temps, mais l'équation d'évolution de $\left\langle S_{z}>\right.$ est couplée à celle de $\left\langle I_{z}\right\rangle$. On en déduit les équations de relaxation à partir d'un état initial d'aimantations électronique et nucléaire connues $\left(<S_{z}>_{0},<I_{z}>_{0}\right)$, dans le cas du rétrécissement extrême :

$$
\begin{aligned}
<S_{z}>=( & \left.<S_{z}>_{0}-\frac{2}{(2 I+1)^{2}-2}<I_{z}>_{0}\right) \mathrm{e}-t / T_{e}^{\prime \prime} \\
& +\frac{2}{(2 I+1)^{2}-2}<I_{z}>_{0} \mathrm{e}^{t / T_{n}^{\prime \prime}}
\end{aligned}
$$




$$
\begin{aligned}
& \left\langle I_{z}\right\rangle=\left\langle I_{z}>{ }_{0} \mathrm{e}^{-t / T_{n}^{\prime \prime},}\right. \\
& \frac{1}{T_{e}^{\prime \prime}}=\sum_{l} 2 k_{l}\left|\alpha_{l}\right|^{2} J(0)=\frac{1}{T_{H}^{\prime \prime}} . \\
& \frac{1}{T_{n}^{\prime \prime}}=\sum_{l} \frac{4}{(2 I+1)^{2}} k_{l}\left|\alpha_{l}\right|^{2} J(0) .
\end{aligned}
$$

Dans ce cas, il existe donc un seul temps de relaxation nucléaire longitudinal $T_{n}^{\prime \prime}$ et 2 temps de relaxation électroniques longitudinaux $T_{e}^{\prime \prime}$ et $T_{n}^{\prime \prime}$; $T_{e}^{\prime \prime}$ ne dépend pas de l'isotope et $T_{n}^{\prime \prime}$ est proportionnel à $(2 I+1)^{2} ; T_{e}^{\prime \prime}$ est plus court que $T_{n}^{\prime \prime}$ dans le rapport $(2 I+1)^{2} / 2$.

On déduit de (46) que l'équation de relaxation de $\left\langle S_{z}\right\rangle$ à partir d'un état initial totalement orienté par de la lumière $\sigma_{+}$ou $\sigma_{-}$est :

pour ${ }^{87} \mathrm{Rb}<S_{z}>= \pm .286 \mathrm{e}^{-t / T_{e}^{\prime \prime}} \pm .214 \mathrm{e}^{-t / 8 T_{e}^{\prime \prime}}$,

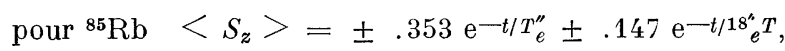

le signe \pm correspond à la condition initiale $<S_{z}>_{0} \equiv \pm .5$.

Il est difficile de confondre les courbes représentatives correspondantes avec une exponentielle unique. Si l'on était conduit à le faire, la constante de temps obtenue dépendrait de la condition initiale à partir de laquelle se fait la relaxation. Nous n'avons pas observé ceci expérimentalement pour la relaxation de ${ }^{87} \mathrm{Rb}$ sur des enduits deutérés. Aussi, pour l'intéraction due a la présence des spins $K$, nous pensons que l'hypothèse

$$
J(\Delta W)=J\left(\omega_{F}\right)=J(0)
$$

n'est pas satisfaite et que par suite $\tau_{\mathrm{c}}>5.10^{-11} \mathrm{sec}$.

$$
\begin{gathered}
3^{\circ} Q=S^{(+1)} \text { et } Q=I^{(+1)} . \\
{\left[\frac{\mathrm{d}}{\mathrm{d} t} \operatorname{Tr}\left\{S^{+1} \sigma_{F+}^{*}\right\}\right]_{2}=-\sum_{l} 2 k_{l}\left|\alpha_{l}\right|^{2} J(\Delta W)} \\
\left(1-\frac{I(2 I+3)}{(2 I+1)^{2}}\right) \operatorname{Tr}\left\{S^{(+1)} \sigma_{F+}^{*}\right\} . \\
{\left[\frac{\mathrm{d}}{\mathrm{d} t} \operatorname{Tr}\left\{S^{+1} \sigma_{F_{-}}^{*}\right\}\right]_{2}=-\sum_{l} 2 k_{l}\left|\alpha_{l}\right|^{2} J(\Delta W)} \\
\left(1-\frac{(I+1)(2 I-1)}{(2 I+1)^{2}}\right) \operatorname{Tr}\left\{S^{(+11} \sigma_{F_{-}}^{*}\right\} .
\end{gathered}
$$

les équations relatives à l'aimantation nucléaire transversale se déduisent des précédentes en substituant dans les 2 membres $I^{(+1)}$ à $S^{(+1)}$.

$\left.<P_{F+} S^{(+1)}\right\rangle^{*}$ et $\left\langle P_{F+} I^{+1}\right\rangle^{*}$ relaxent avec la même constante de temps $\left(T_{2}\right)_{F+}^{\prime \prime}$.

$\left.<P_{F-} S^{\complement+1)}\right\rangle^{*}$ et $\left\langle P_{F-} I^{+1}\right\rangle^{*}$ relaxent avec la même constante de temps $\left(T_{2}\right)_{F-1}^{\prime \prime}$. Mais contrairement à ce qui se passe lorsque $J(\Delta W)=0$, dans le cas du rétrécissement extrême, ces 2 constantes de temps sont différentes:

$$
\begin{aligned}
& \left(\frac{1}{T_{2}^{\prime \prime}}\right)_{F_{+}}=\sum_{l} 2 k_{l}\left|\alpha_{l}\right|^{2} J(0)\left(1-\frac{I(2 I+3)}{(2 I+1)^{2}}\right), \\
& \left(\frac{1}{T_{2}^{\prime \prime}}\right)_{F_{-}}=\sum_{l} 2 k_{l}\left|\alpha_{l}\right|^{2} J(0)\left(1-\frac{(I+1)(2 I-1)}{(2 I+1)^{2}}\right) .
\end{aligned}
$$

On trouve que dans le cas de ${ }^{87} \mathrm{Rb}(I=3 / 2)$ $\frac{\left(T_{2}\right)_{F-}^{\prime \prime}}{\left(T_{2}\right)_{F+}^{\prime \prime}}=.63$ et dans le cas de

$$
{ }^{85} \mathrm{Rb}(I=5 / 2) \frac{\left(T_{z}^{\prime \prime}\right)_{F-}}{\left(T_{z}^{\prime \prime}\right)_{F+}}=.73
$$

Alors que pour $J(\Delta W)=0$ on avait

$$
\frac{T_{n}^{\prime}}{T_{2}^{\prime}}=\frac{T_{1}^{\prime}}{T_{2}^{\prime}}=1,
$$

maintenant on trouve :

$$
\begin{aligned}
\frac{T_{n}^{\prime \prime}}{\left(T_{2}^{\prime \prime}\right)_{F+}} & =\frac{2 I^{2}+I+1}{2}\left(=7 / 2 \text { pour }{ }^{87} \mathrm{Rb}\right) \\
\text { et } \quad \frac{T_{n}^{\prime \prime}}{\left(T_{2}^{\prime \prime \prime}\right)_{F-}} & =\frac{2 I^{2}+3 I+2}{2}\left(=11 / 2 \text { pour }{ }^{87} \mathrm{Rb}\right) .
\end{aligned}
$$

La valeur de ce rapport peut donc en principe permettre de déterminer si nous sommes dans le cas $J(\Delta W)=0$ ou le cas du rétrécissement extrême.

C) Résultats Dans Le cas Général : $J(\Delta W) / J(0)$ QUELCONQUE. - En combinant les résultats des paragraphes $(\mathrm{A})$ et $(\mathrm{B})$ précédents, on arrive aux conclusions suivantes :

$$
\begin{aligned}
& 1^{0} \quad Q=S . I \\
& \left.\frac{\mathrm{d}}{\mathrm{d} t}\langle\delta . \boldsymbol{I}\rangle=-\sum_{l} 2 k_{l}\left|\alpha_{l}\right|^{2} J(\Delta W)<\boldsymbol{s . \boldsymbol { I }}\right\rangle .
\end{aligned}
$$

De façon générale, il n'existe qu'un seul temps de relaxation hyperfin $T_{H}$.

$$
1 / T_{\mathrm{H}}=\sum_{l} 2 k_{l}\left|\alpha_{l}\right|^{2} J(\Delta W) .
$$

$T_{H}$ ne varie d'un isotope à l'autre que par l'intermédiaire de l'écart hyperfin. Il est indépendant du spin nucléaire.

$$
\begin{aligned}
& 2^{\circ} Q= S_{z} \text { et } Q=I_{z} . \\
& \frac{\mathrm{d}}{\mathrm{d} t}<S_{z}>=-\sum_{l} 2 k_{l}\left|\alpha_{l}\right|^{2}\left\{\left[\frac{J\left(\omega_{F}\right)}{(2 I+1)^{2}}\right.\right. \\
&\left.+\left(1-\frac{1}{(2 I+1)^{2}}\right) J(\Delta W)\right]<S_{z}> \\
&\left.\quad-\frac{2 J(\Delta W)}{(2 I+1)^{2}}<I_{z}>\right\} . \\
& \frac{\mathrm{d}}{\mathrm{d} t}<I_{z}>=-\sum_{1} 2 k_{l}\left|\alpha_{l}\right|^{2} \frac{J\left(\omega_{F}\right)+J(\Delta W)}{(2 I+1)^{2}}<I_{z}>.
\end{aligned}
$$

Les équations de relaxation à partir d'un état initial d'aimantation électronique et nucléaire connues $\left(<S_{z}>_{0},<I_{z}>_{0}\right)$ sont done :

$$
\begin{gathered}
\begin{array}{c}
\left\langle S_{z}\right\rangle=\left(\left\langle S_{z}>_{0}-\frac{2}{(2 I+1)^{2}-2}<I_{z}>_{0}\right) \mathrm{e}^{-t / T_{e}}\right. \\
\quad+\frac{2}{(2 I+1)^{2}-2}<I_{z}>_{0} \mathrm{e}^{-t / T_{n}}
\end{array} \\
\left\langle I_{z}\right\rangle=\left\langle I_{z}>_{0} \mathrm{e}^{-t / T_{n}}\right.
\end{gathered}
$$


avec

$$
\begin{aligned}
& \frac{1}{T_{e}}=\sum_{l} 2 k_{l}\left|\alpha_{l}\right|^{2}\left[\frac{J\left(\omega_{F}\right)-J(\Delta W)}{(2 I+1)^{2}}+J(\Delta W)\right] . \\
& \frac{1}{T_{n}}=\sum_{l} 2 k_{l}\left|\alpha_{l}\right|^{2} \frac{J\left(\omega_{F}\right)+J(\Delta W)}{(2 I+1)^{2}} .
\end{aligned}
$$

(Si on fait dans ces équations $\Delta W=0$, et $I=0$, on retrouve pour $<S_{z}>$ la même équation d'évolution que pour un spin $S$ isolé (éq. 37 bis).

Dans tous les cas $<I_{z}>$ relaxe avec une seule constante de temps $T_{n}$ (éq. 58), $T_{n}$ varie d'un isotope à l'autre par l'intermédiaire de l'écart hyperfin $(J(\Delta W))$ et surtout par l'intermédiaire de la multiplicité du spin $I: T_{n}$ est proportionnel à $(2 I+1)^{2}$. Remarquons que la détermination expérimentale du rapport $T_{n} / T_{H}$ en champ faible serait un très bon critère pour la comparaison de $J(\Delta W)$ à $J(0)$ :

$$
\frac{T_{H}}{T_{n}}=(2 I+1)^{2} \frac{J(\Delta W)}{J(0)+J(\Delta W)}=\frac{(2 I+1)^{2}}{2+\Delta W^{2} \tau_{\mathrm{c}}^{2}} .
$$

C'est même une méthode de mesure de $\tau_{c}$ qui pourrait être précise dans le cas où $\Delta W \tau_{\mathrm{c}}>1$.

$<S_{z}>$ relaxe en général avec 2 constantes de temps $T_{e}$ et $T_{n}$ (éq. 57 et 58 ). Nous avons déjà vu que :

๙) Si $J(\Delta W)=J\left(\omega_{F}\right)=J(0)$ la courbe de relaxation diffère nettement d'une exponentielle.

乃) $\mathrm{Au}$ contraire, si $(2 I+1)^{2} J(\Delta W) \ll J\left(\omega_{\mathrm{F}}\right)$ les 2 constantes de temps sont pratiquement égales et la courbe de relaxation est une exponentielle.

Dans le cas intermédiaire, c'est-à-dire pour des valeurs de $\tau_{c}$ de l'ordre de quelques $10^{-10}$ sec., $T_{e}$ et $T_{n}$ sont dans un rapport compris entre 1 et $(2 I+1)^{2} / 2$; on trouve que la combinaison linéaire de ces 2 exponentielles peut être facilement confondue avec une exponentielle unique. On peut aussi montrer que le rapport des " temps de relaxation » pour les 2 isotopes ${ }^{85} \mathrm{Rb}$ et ${ }^{87} \mathrm{Rb}$ est inférieur à 2.25. Il n'est pas exclu que l'on se trouve en pratique dans ce cas.

$$
\begin{aligned}
& 3^{\circ} \quad Q=S^{(+1)} \text { et } Q=I^{(+1)} \text {. } \\
& \left\{\begin{array}{l}
\frac{\mathrm{d}}{\mathrm{d} t}<P_{F+} S^{(+1)}>*=-\frac{1}{\left(T_{2}\right)_{F+}}<P_{F_{+}} S^{(+1)}>* \\
\frac{\mathrm{d}}{\mathrm{d} t}<P_{F_{-}} S^{(+1)}>*=-\frac{1}{\left(T_{2}\right)_{F-}}<P_{F_{-}} S^{(+1)}>* .
\end{array}\right.
\end{aligned}
$$

et pour $I^{(+1)}$ les équations analogues obtenues en substituant dans les 2 membres $I^{(+1)}$ à $S^{(+1)}$.

Avec l'approximation $J\left(\omega_{K}\right)=J(0),\left(T_{2}\right)_{F+}$ et $\left(T_{2}\right)_{F}$ - sont donrées par les expressions :

$$
\begin{aligned}
\frac{1}{\left(T_{2}\right)_{F}+}=\sum_{l} 2 k_{l}|\alpha l|^{2}\{ & \frac{J(0)+J\left(\omega_{F}\right)-2 J(\Delta W)}{2(2 I+1)^{2}} \\
& \left.+\left(1-\frac{I(2 I+3)}{(2 I+1)^{2}}\right) J(\Delta W)\right\}
\end{aligned}
$$

$$
\begin{aligned}
\frac{1}{\left(T_{2}\right)_{F-}}=\sum_{l} 2 k_{l}\left|\alpha_{l}\right|^{2}\left\{\frac{J(0)+J\left(\omega_{F}\right)-2 J(\Delta W)}{2(2 I+1)^{2}}\right. \\
\left.+\left(1-\frac{(I+1)(2 I-1)}{(2 I+1)^{2}}\right) J(\Delta W)\right\}
\end{aligned}
$$

Si on fait dans ces équations $\Delta W=0, I=0$ (et par conséquent $P_{F_{+}}=1$ et $P_{F_{-}}=0$ ), on retrouve pour $\left\langle S^{+1}\right\rangle *$ la même équation d'évolution que pour un spin $S$ isolé :

avec

$$
\frac{\mathrm{d}}{\mathrm{d} t}<S+1>*=-\frac{1}{T_{S_{2}}}<S+1>*
$$

$$
\frac{1}{T_{S 2}}=\sum_{l} 2 k_{l}\left|\alpha_{l}\right|^{2}\left(\frac{1}{2} J(0)+{ }_{2}^{1} J\left(\omega_{S}\right)\right)
$$

Rappelons que la condition de validité du calcul de $T_{2}: H_{0}<\bar{H}_{0}$ restreint cette étude à un domaine de champ très faible. Expérimentalement, nous avons opéré jusqu'ici dans un champ $H_{0}>\vec{H}_{0}$, cas que nous envisageons dans la $3^{\mathrm{e}}$ partie.

En comparant les équations (57), (58), (61) et (62), on constate que l'on a en général en champ faible $\left(J\left(\omega_{F}\right)=J(0)\right) T_{n}>\left(T_{2 F^{+}}>\left(T_{2}\right)_{F}{ }^{-}>T_{e}\right.$ c'est-à-dire que malgré le caractère isotrope du hamiltonien $\mathcal{H}_{1}(t)$, la relaxation $\mathrm{du}$ spin $S$ ne se fait pas en général de façon isotrope : les aimantations longitudinale et transversale relaxent de façon différente. On pouvait s'attendre à ce résultat car une transition d'un niveau F à l'autre sous l'effet de la relaxation peut conserver partiellement le moment angulaire suivant $H_{0}$; par contre elle détruit complètement le moment angulaire transversal parce que le mouvement propre de ce moment est différent dans les 2 niveaux $F$. (Facteurs de Landé opposés.)

Nous verrons que le seul cas où la relaxation de $S$ se fait de façon isotrope est celui où

$$
(2 I+1)^{2} J(\Delta W) \ll J\left(\omega_{F}\right) ;
$$

(les équations (57) et (58) permettent de montrer que dans cette hypothèse $T_{e} \simeq T_{n}$ et les équations (61) et (62) que $\left.\left(T_{2}\right)_{F+} \simeq\left(T_{2}\right)_{F-} \simeq T_{n}\right)$. Le temps de relaxation hyperfin $T_{H}$ est alors très long devant tous les autres (éq. 51). Nous pouvons séparer les atomes en 2 classes : ceux qui sont dans $F_{+}$et ceux qui sont dans $F_{-}$; la relaxation n'est pas susceptible de faire passer les atomes d'une classe à l'autre. Le problème devient celui de la relaxation de 2 catégories de spins $F$ soumis aux hamiltoniens Zeeman et de perturbation. On peut montrer, par un raisonnement analogue à celui fait dans la référence 3 (p. 292), que pour chacune de ces 2 classes, la relaxation est isotrope, c'est-à-dire $\left(T_{1}^{\prime}\right)_{F_{+}}=\left(T_{2}^{\prime}\right)_{F_{+}}$et $\left(T_{1}^{\prime}\right)_{F_{-}}=\left(T_{2}^{\prime}\right)_{F_{-}}$. Notre calcul montre que, de plus, $\left(T_{1}^{\prime}\right)_{F_{+}}=\left(T_{1}^{\prime}\right)_{F_{-}}=T_{1}^{\prime}$. Ce résultat est une conséquence du fait que les facteurs de Landé des niveaux $F_{+}$et $F_{-}$ont même valeur absolue. 
D) Conclusions sur la relaxation produite PAR LES SPINS DE LA ParoI. - Nous verrons que toutes les remarques que nous avons faites dans les parties (A), (B) et (C) sont généralisables à toute interaction $\mathcal{H}_{1}(t)$ n'agissant que sur $S$. (Nous démontrons dans la $4^{\mathrm{e}}$ partie que les équations d'évolution des observables sont les mêmes à condition de définir différemment les coefficients $k_{l}$ et $\left|\alpha_{l}\right|^{2}$.) Nous faisons maintenant au contraire des remarques qui concernent uniquement l'interaction due aus spins $K$ de la paroi (qui peut être à la fois scalaire et tensorielle).

1) La théorie permet de prévoir que les temps de relaxation sur deux parois chimiquement identiques (même temps de séjour, même temps de corrélation), mais différant par le moment magnétique et la multiplicité des spins $K$, sont dans un rapport déterminé.

Nous avons vu que pour l'interaction scalaire :

$$
2 k_{0}\left|\alpha_{0}\right|^{2}=\frac{64}{27} \pi^{2} K(K+1) \frac{\gamma_{S}^{2} \gamma_{K}^{2}}{R_{0}^{6}} \hbar^{2} \frac{\tau_{S}}{\tau_{S}+\tau_{V}},
$$

et que pour l'interaction tensorielle :

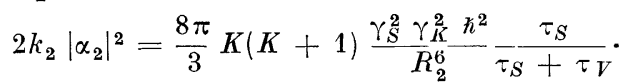

L'effet est donc le même quel que soit le type de l'interaction, scalaire ou tensorielle. Le rapport prévu du coefficient $\gamma_{K}^{2} K(K+1)$ entre paraffines hydrogénées et deutérées est 16. Le rapport observé expérimentalement pour des paraffines à longue chaîne n'étant que de l'ordre de 6 , nous pouvons dire qu'il existe une seconde interaction ne dépendant pas des spins $K$, qui est de peu d'importance pour les enduits hydrogénés et qui, dans le cas des enduits deutérés, est du même ordre de grandeur que l'interaction que nous envisageons dans le présent mémoire $\left(^{3}\right)$.

2) Pour deux parois chimiquement diflérentes, les facteurs susceptibles de modifier le temps de relaxation sont :

- le temps de séjour et le temps de corrélation. Il semble peu probable que le temps de relaxation des spins $K$ eux-mêmes (long devant $\tau_{s}$ ) puisse intervenir ;

(3) L'observation expérimentale de deux constantes de temps pour la relaxation de $\left\langle S_{z}\right\rangle$ sur des enduits deutérés permet de conclure que le temps de corrélation associé à cette seconde intéraction est court (de l'ordre de quelques $10^{12} \mathrm{~s}$.)
- les valeurs des moments magnétiques $\mu_{K}$ et des spins nucléaires $K$ de la paroi ;

- la distance moyenne d'approche des atomes de Rubidium vis-à-vis des noyaux de l'enduit porteurs d'un moment magnétique $\mu_{K}$.

Notons qu'un allongement de $T_{1}$ a été signalé (réf. 8) dans le cas de la relaxation de l'hydrogène atomique quand on substitue des parois de Téflon $\left(\mathrm{CF}_{2}\right)_{n}$ à des parois de paraffine $\left(\mathrm{CH}_{2}\right)_{n}$. Les atomes d'hydrogène et de Fluor ont le même spin et des moments magnétiques nucléaires très voisins ; la polarisabilité du fluor est un peu supérieure à celle de l'hydrogène et par suite, la durée de l'interaction est probablement plus longue sur un enduit de téflon que sur un enduit paraffiné ; par contre le rayon atomique du fluor est nettement plus grand que celui de l'hydrogène, donc la force de l'interaction et $1 / T_{1}^{\prime}$, proportionnels à $1 /\left|r_{S K}(t)\right|^{6}$, sont beaucoup plus faibles. Ceci permet peut-être d'expliquer l'avantage d'un enduit de téflon sur un enduit de paraffine hydrogénée dans les expériences de Ramsey et al. [8]. Malheureusement, un enduit de téflon ne peut pas être utilisé avec les vapeurs alcalines qui l'attaquent chimiquement.

3) Pour un enduit donné, l'importance relative des interactions scalaire et tensorielle est difficile à évaluer ; en outre leurs temps de corrélation sont peut-être différents. La définition de $R_{2}$,

$$
1 /\left(R_{2}\right)^{6}=1 \widetilde{\left|r_{S K}(t)\right|^{6}}
$$

ne permet pas de relier ce paramètre simplement à la distance moyenne d'adsorption des atomes de Rubidium sur la surface. (Notons par exemple que $R_{2}$ varie en sens inverse de la densité des spins $K$ sur la surface.) De même, le calcul de $R_{0}$, $\frac{1}{\left(R_{0}\right)^{6}}=\tilde{\left|\delta_{r_{S i}}^{3}(t)\right|^{2}}$, est très sensible à la déformation des fonctions d'onde que peut produire l'interaction qui détermine le temps de séjour sur la paroi. Notons seulement qu'à partir des valeurs expérimentales obtenues pour $T_{1}$ et $\tau_{\mathrm{c}}$ et en faisant l'hypothèse $\tau_{\mathrm{s}}=\tau_{c}$, on peut évaluer $R_{2}$. Avec $T_{1}=0,2 \mathrm{~s}, \tau_{\mathrm{c}}=\tau_{\mathrm{c}}=10^{-10} \mathrm{~s}$, $\tau_{\mathrm{V}}=2 \times 10^{-4} \mathrm{~s}$, on calcule $R_{2}=1 \AA$ en supposant que la relaxation est déterminée par l'interaction dipole-dipole seule. On trouve pour $R_{0}$ une valeur du même ordre en supposant que l'interaction scalaire agit seule.

Manuscrit reçu le 15 février 1963.

\section{BIBLIOGRAPHIE}

[1] Bouchiat (M. A.) et Brossel (J.), C. R. Acad. Sc., 1962, 254, 3650.

[2] Bouchiat (M. A.) et Brossel (J.), C. R. Acad. Sc., 1962, 254, 3828.

[3] Abragam (A.), The Principles of Nuclear Magnetism, Oxford University Press, chap. VIII.

[4] Bernhein (R.), J. Chem. Physics, 1962, 36, 135.

[5] Bethe et Salpeter, Handbuch der Physik, XXXV, Atome I, p. 193.

[6] Raysay (N.F.), Molecular beams, Chapter III. p. 8't.

[7] Barrat (J. P.) et Cohex-Taxioldji (C.), J. Physique Rad., 1961, 22, 329 et $\mathbf{4} 43$.
[8] Kleppner (D.), Goldenberg (H. M.) et Ramay (N. F.), Phys. Rev., 1962, 126, 603 .

[9] Edronds (A. R.), Angular Momentum in Quantum Electronics, Princeton Lniversity Press, chapitre 7.

[10] Cagnac (B.) et Brossel (J.), C. R. Acad. Sc., 1959, $249,253$.

[11] Barral (S.), Diplôme d'Études Supérieures, Paris, 1963.

[12] Cagrac (B.), Thèse, Paris, 1960 ; Ann. Physique, 1961, 6,467 .

[13] Brossel (J.), Quantum Electronics, Columbia University Press, New York, 1961, p. 95. 